\title{
Comparing Oncotype DX Recurrence Score Categories with Immunohistochemically Defined Luminal Subtypes
}

\author{
Yoshio Mizuno ${ }^{1}$, Hiromi Fuchikami1, Naoko Takeda',2, Junichi Yamada ${ }^{3}$, Yuko Inoue², \\ Hiroshi Seto ${ }^{4}$, Kazuhiko Sato ${ }^{1}$ \\ ${ }^{1}$ Department of Breast Oncology, Tokyo-West Tokushukai Hospital, Tokyo, Japan \\ ${ }^{2}$ Inoue Ladies Clinic, Tokyo, Japan \\ ${ }^{3}$ Department of Clinical Pathology, Tokyo-West Tokushukai Hospital, Tokyo, Japan \\ ${ }^{4}$ Seto Hospital, Saitama, Japan \\ Email: "twbreast@yahoo.co.jp
}

Received 9 December 2015; accepted 19 March 2016; published 22 March 2016

Copyright (C) 2016 by authors and Scientific Research Publishing Inc.

This work is licensed under the Creative Commons Attribution International License (CC BY). http://creativecommons.org/licenses/by/4.0/

(c)

\begin{abstract}
Background: At the 13th St Gallen International Breast Cancer Conference in 2013, a new definition of luminal A-like and luminal B-like breast cancer was proposed, involving the expression of estrogen receptor (ER), progesterone receptor (PgR), and Ki-67. We examined the rate of concordance between the risk groups using the Oncotype DX recurrence score (RS) and the previous and newly proposed luminal subtypes with the standardized Ki-67 assessment. Method: The relationship between a previously and newly proposed, immunohistochemically defined luminal A and B subtype with the Oncotype DX RS of 41 cases of T1-2 N0-1 MO (ER positive, HER2 negative) breast cancer was assessed. We first classified the patients into the previously defined luminal $A$ and $B$ subtypes, according to the level of Ki-67 as either "low" ( $<14 \%)$ or "high" $(\geq 14 \%)$, as assessed by local pathologists. Next, to consider the necessity for standardizing Ki-67 measurement methods, we re-examined Ki-67 with a central review. By introducing PgR positivity ( $\geq 20 \%$ ), we classified these patients to newly proposed luminal subtypes and compared them with the risk groups stratified by Oncotype DX RS. Results: In the previously proposed luminal subtypes, the concordance rate between luminal $A$ and the low RS category was $76.5 \%$ according to local pathologists and $\mathbf{9 0 . 1 \%}$ by central review, whereas the rate between luminal $B$ and the intermediate to high $\mathrm{RS}$ category was $46.7 \%$ and $45.8 \%$, respectively. In newly proposed luminal subtypes, the concordance rate between luminal $A$ and low RS category was $100 \%$ and between the luminal $B$ and intermediate to high RS category was $53.6 \%$. Conclusions: Although this study was based on a retrospective chart review of a small sample size, the newly proposed luminal subtypes including
\end{abstract}

\footnotetext{
"Corresponding author.
} 
addition of PgR positivity with the standardized Ki-67 assessment appeared to improve the concordance rate especially between luminal A and low RS category.

\author{
Keywords
}

Breast Cancer, Oncotype DX, Ki-67, Standardized Assessment

\title{
1. Introduction
}

Breast cancer is a clinically and biologically heterogeneous disease. Around $80 \%$ of breast cancer patients have hormone receptor positive disease, and these patients receive endocrine therapy to improve their prognosis [1]. Determining in which patients cytotoxic chemotherapy can be avoided has become a major problem. The identification of intrinsic subtypes using molecular technologies is the most precise assay to identify low or high risk patients; however, where such assays are unavailable, surrogate definitions of subtype can be obtained by IHC measurements of ER, PgR, Ki-67, and HER2 status [2].

Ki-67 is widely available as a prognostic factor and a predictive marker of chemotherapy benefit in breast cancer patients [3]-[8]. Ki-67 is frequently used as a key marker to guide systemic treatment decisions in both the neoadjuvant and adjuvant settings, although there is no standardized method with good reproducibility and reliable cutoff values [9]. In preanalytic validity, formalin fixation adversely affects the measurement results. Similarly, in terms of analytical validity, tumor heterogeneity and the lack of a standard measuring method by pathologists may also affect measurement of Ki-67 [10].

The Oncotype DX test (Genomic Health, Redwood City, CA, USA) is a multi-gene reverse transcriptase PCR (RT-PCR) assay that was developed for use with formalin-fixed paraffin-embedded tumors, and provides the Recurrence Score $^{\circledR}$ (RS) that predicts chemotherapy benefit and the risk of distant breast cancer recurrence [11]-[16]. Based on RS, patients are divided into three risk groups: low risk $(\mathrm{RS}<18)$, intermediate risk $(18 \leq$ $\mathrm{RS}<31$ ), or high risk (RS $\geq 31$ ). The use of RS to guide clinical treatment decisions has been incorporated into clinical guidelines such as ASCO [17], NCCN [18], ESMO [19], and the St Gallen Consensus Guidelines [20] [21].

Furthermore, research in recent years has shown that PgR should be included when considering chemotherapy, with a PgR cut-off of $<20 \%$ argued for inclusion in chemotherapy [22]. At the 13th St Gallen International Breast Cancer Conference in 2013, to distinguish between luminal A-like and luminal B-like breast cancer, a new definition was proposed, involving a combination of the expression of ER, PgR, and Ki-67.

Because too many variations in analytical practice restrict the value for the clinical utility of Ki-67 [10] [23] [24], the Oncotype DX test has often been introduced as a reliable biomarker test, although it is very expensive and the National Health Insurance Program in Japan does not cover its cost. Accordingly, we need to more efficiently select low or intermediate to high RS patients. To consider the possibility of selecting patient with a low or an intermediate to high RS more accurately, we hypothesize that the newly proposed definition of luminal subtypes at the 13th St Gallen International Breast Cancer Conference and the standardized Ki-67 assessment are able to find the low or high risk patients. Here we examined the rate of concordance between risk groups from the Oncotype DX RS and the previous and newly proposed luminal A and B subtypes with the standardized Ki-67 assessment. Furthermore, we compared the correlation of the Oncotype DX RS with histopathological characteristics of the tumor including PgR status and Ki-67 in these patients.

\section{Methods}

\subsection{Patients and Materials}

The cohort included consecutive patients with early breast cancer who underwent surgery from December 2011 to January 2015 at the Tokyo-West Tokushukai Hospital (Tokyo, Japan) and who requested RS. Of these patients, the Oncotype DX test was performed in 41 patients with early breast cancer (T1-2, N0-1, M0, ER-positive, and HER2-negative). The Oncotype DX test is intended to be used by patients with newly diagnosed, earlystage (stage I or II), lymph node-negative, ER-positive, and HER2-negative invasive breast cancer who will be 
treated with adjuvant therapy. The Oncotype DX test may also be used to select postmenopausal patients with stage II or IIIa, lymph node-positive, ER-positive, and HER2-negative (and those who will be treated with hormone therapy) invasive breast cancer.

\subsection{Assessing Ki-67 Scores and Other Biomarkers}

An IHC score consisting of at least $10 \%$ positive cells was used to define ER/PgR positivity. A positive score for HER2 was either HER2 3+ by IHC analysis (defined as uniform intense membrane staining of $>30 \%$ of invasive tumor cells) or fluorescence in situ hybridization (ratio of HER2 to chromosome 17 centromere of >2.0). Immunohistochemical staining was quantitatively evaluated by light microscopy, in which the entire tissue section was scanned at low-power magnification $(10 \times)$ to determine areas with the highest number of positive nuclei (hot-spots) within the invasive component. Ki-67 was expressed as the percentage of cells positive for mindbomb E3 ubiquitin protein ligase 1 (MIB-1) among a total of at least 1000 malignant cells at high-power magnification $(40 \times)$. Nuclear staining of the tumor cells was considered as negative if $14 \%$ or fewer of the cells were stained for Ki-67 and as positive if more than 14\% were stained for KI-67. An MIB-1 clone (Dako, Carpinteria, CA, USA) was used for immunohistochemical analysis of Ki-67. All cases were evaluated by many registered local pathologists. Although many different systems for grading pathological responses have been proposed, no standard method has yet been adopted.

A central review by a professionally trained physician was performed by scanning magnification to count at least 1000 cells in the most densely labeled areas. For all nonmatching cases, the percentage of tumor cells with any nuclear staining was recorded. The central review used calculations based on the hot-spot counting method, because counting the area with the highest number of positive cells was more reproducible than random counting.

\subsection{The Relationship of the Previous and Newly Proposed Immunohistochemically Defined Luminal Subtypes Compared with the Oncotype DX RS}

To assess the validity of the previous and newly proposed surrogate definitions of the "luminal A" and "luminal B (HER2-negative)" intrinsic molecular subtypes of breast cancer outlined at the 2011 and 2013 St Gallen International Breast Cancer Conference, we hypothesized that the newly proposed definition of luminal subtypes improves the precision of selecting patients with intermediate to high RS. We also considered the necessity of standardizing Ki-67 measurement methods by central review. For that reason, we examined the rate of concordance between the risk groups using the Oncotype DX RS and the previously defined and newly proposed luminal subtypes with the standardized Ki-67 assessment. We first classified these 41 patients into the previously proposed luminal A and B subtypes according to the level of Ki-67 with either a "low" $(<14 \%)$ or "high" $(\geq 14 \%)$ core as assessed by local pathologists. Next, to consider the necessity of standardizing Ki-67 measurement methods, we re-examined Ki-67 status by central review. Finally, by including PgR positivity ( $\geq 20 \%$ ), we also classified patients who had their Ki-67 status re-examined by central review to newly proposed luminal subtypes, and compared them with the risk groups as per Oncotype DX RS.

\subsection{The Correlation of the Oncotype DX RS with PgR Status and Ki-67}

The ystat 2008 Software (statistical program file developed with Microsoft Excel, Igakutoshoshuppan, Tokyo, Japan) was used for Spearman's correlation analysis.

According to the policies of our institutional ethics committee, general consent was obtained from all patients receiving medical care.

\section{Results}

\subsection{Baseline Clinical Characteristics}

In this study, 41 patients with early breast cancer, who were eligible for the Oncotype DX test, were identified. These patients were divided into two groups according to Oncotype DX RS (i.e., RS $<18$ vs. $18 \leq \mathrm{RS}$ ). A total of $26(63.4 \%)$ of 41 patients had a RS < 18, whereas 15 (36.6\%) of the 41 patients had a RS of 18 or more. The distribution of clinicopathological features in both groups is shown in Table 1. The median patient age was 54.6 
Table 1. Baseline clinicopathological characteristics.

\begin{tabular}{|c|c|c|c|}
\hline Characteristics & $\begin{array}{c}\text { All patients } \\
\mathrm{n}=41 \\
\text { No. }(\%)\end{array}$ & $\begin{array}{c}\mathrm{RS}<18 \\
\mathrm{n}=26 \\
\text { No. }(\%)\end{array}$ & $\begin{array}{c}18 \leq \mathrm{RS} \\
\mathrm{n}=15 \\
\text { No. }(\%)\end{array}$ \\
\hline Median age, year (range) & $54.6(28-80)$ & $51.8(28$ - 77) & $59.7(43-80)$ \\
\hline \multicolumn{4}{|l|}{ Menopause } \\
\hline Yes & $21(51.2)$ & $10(38.5)$ & $11(73.3)$ \\
\hline No & $20(48.8)$ & $16(61.5)$ & $4(26.7)$ \\
\hline \multicolumn{4}{|l|}{ Surgery } \\
\hline Mastectomy & $8(19.5)$ & $4(15.4)$ & $4(26.7)$ \\
\hline Breast conserving surgery & $33(80.5)$ & $22(84.6)$ & $11(73.3)$ \\
\hline \multicolumn{4}{|l|}{ Tumor size } \\
\hline $\mathrm{T}<2 \mathrm{~cm}$ & $30(73.2)$ & $19(73.1)$ & $11(73.3)$ \\
\hline $2 \mathrm{~cm} \leq \mathrm{T}<5 \mathrm{~cm}$ & $9(22.0)$ & $6(23.1)$ & $3(20.0)$ \\
\hline $5 \mathrm{~cm} \leq \mathrm{T}$ & $2(4.8)$ & $1(3.8)$ & $1(6.7)$ \\
\hline \multicolumn{4}{|l|}{ Node status } \\
\hline 0 & 38 (92.7) & 25 (96.2) & $13(86.7)$ \\
\hline $1-3$ & $3(7.3)$ & $1(3.8)$ & $2(13.3)$ \\
\hline 4- & 0 & 0 & 0 \\
\hline \multicolumn{4}{|l|}{ PgR status } \\
\hline $\operatorname{PgR}<20 \%$ & $9(22.0)$ & $1(3.8)$ & $8(53.3)$ \\
\hline $20 \% \leq \operatorname{PgR}$ & $32(78.0)$ & 25 (96.2) & $7(46.7)$ \\
\hline \multicolumn{4}{|l|}{ Ki-67 (local) } \\
\hline Ki-67 < 14\% & $11(26.8)$ & $8(30.8)$ & $3(20.0)$ \\
\hline $14 \% \leq \mathrm{Ki}-67$ & $30(73.2)$ & $18(69.2)$ & $12(80.0)$ \\
\hline \multicolumn{4}{|l|}{ Ki-67 (central) } \\
\hline Ki-67 < 14\% & $17(41.5)$ & $13(50.0)$ & $4(26.7)$ \\
\hline $14 \% \leq \mathrm{Ki}-67$ & $24(58.5)$ & $13(50.0)$ & $11(73.3)$ \\
\hline
\end{tabular}

years (range, 28 - 80 years) with 20 premenopausal and 21 postmenopausal. A total of 33 (80.5\%) patients had breast conserving surgery. The median tumor size was $1.54 \mathrm{~cm}$ (range, $0.3-7.0 \mathrm{~cm}$ ).

\subsection{Comparing Risk Groups Using Oncotype DX RS and the Previously Proposed Luminal Subtypes}

According to the level of Ki-67 assessed by local pathologists in the previously proposed luminal subtypes, 11 patients were categorized into the luminal A subtype, whereas 30 were categorized into the luminal B subtype. Among the luminal A subtype, 8 of 11 cases were identified as low risk. Among the luminal B subtype, 12 of 30 cases were identified as being at an intermediate to high risk. As assessed by local pathologists, the concordance rate between the luminal A and low risk group was 72.7\% (8/11) and that between the luminal B and intermediate to high risk group was $40 \%$ (12/30) (Table 2).

According to the level of Ki-67 assessed by central review in the previously proposed luminal subtypes, 17 patients were categorized into the luminal A subtype and 24 patients were categorized into the luminal B subtype. Among the luminal A subtype, 13 of 17 cases were identified in the low risk group. Among the luminal B subtype, 11 of 24 cases were included in the intermediate to high risk group. As assessed by local pathologists, the concordance rate between the luminal A and low risk group was $76.5 \%$ (13/17), whereas the concordance 
rate between the luminal B and intermediate to high risk group was 45.8\% (11/24) (Table 3).

\subsection{Comparing Risk Groups Using Oncotype DX RS and Newly Proposed Luminal A and B Subtypes}

Among patients who had their Ki-67 status re-examined by central review and according to the newly proposed luminal subtypes, 13 patients were categorized into the luminal A subtype, and the remaining 28 patients were categorized into the luminal B subtype. Among the luminal A subtype, all of the 13 cases were included in the low risk group. Among luminal B subtype, 15 of 28 cases were included in the intermediate to high risk group. The concordance rate between luminal A and low risk group were $100 \%(13 / 13)$ as determined by the central review, whereas the concordance rate between luminal B and the intermediate to high risk group was 53.6\% (15/28) (Table 4). We found that 53.6\% (15 patients) who were identified in the intermediate to high risk group were considered for adjuvant chemotherapy in our cohort. When introducing the newly proposed luminal subtypes and Ki-67 status as assessed by the central review to order the Oncotype DX test, the concordance rate between the luminal A and low risk group, and the luminal B and intermediate to high risk group improved from $72.7 \%$ to $100 \%$ and $40.0 \%$ to $53.6 \%$, respectively.

\subsection{The Correlation between Oncotype DX RS with Ki-67 and PgR Status}

We also analyzed the correlation between other histopathological characteristics of the tumor with Oncotype DX RS. In our study, there was a significant correlation between PgR status and Oncotype RS (correlation coefficient $=0.50, \mathrm{P}<0.01$; Figure 1). However, there was no significant correlation between Ki-67 status as assessed by either a local pathologist or central review and the Oncotype RS (correlation coefficient $=0.08, \mathrm{P}=$ 0.30 ; correlation coefficient $=0.09, \mathrm{P}=0.28$, respectively; Figure 2, Figure 3 ).

Table 2. Comparison between the risk groups as per Oncotype DX RS and previously proposed luminal subtypes according to the level of Ki-67 assessed by local pathologists.

\begin{tabular}{cccc}
\hline & RS low risk & RS int.-high risk & Total \\
\hline Luminal A & 8 & 3 & 11 \\
Luminal B & 18 & 12 & 30 \\
Total & 26 & 15 & 41 \\
\hline
\end{tabular}

Table 3. Comparison between the risk groups as per Oncotype DX RS and previously proposed luminal subtypes according to the level of Ki-67 assessed by central review.

\begin{tabular}{cccc}
\hline & RS low risk & RS int.-high risk & Total \\
\hline Luminal A & 13 & 4 & 17 \\
Luminal B & 13 & 11 & 24 \\
Total & 26 & 15 & 41 \\
\hline
\end{tabular}

Table 4. Comparison between the risk groups as per Oncotype DX RS and newly proposed luminal A and B subtypes with the level of Ki-67 assessed by central review.

\begin{tabular}{cccc} 
& RS low risk & RS int.-high risk & Total \\
\hline Luminal A & 13 & 0 & 13 \\
Luminal B & 13 & 15 & 28 \\
Total & 26 & 15 & 41 \\
\hline
\end{tabular}




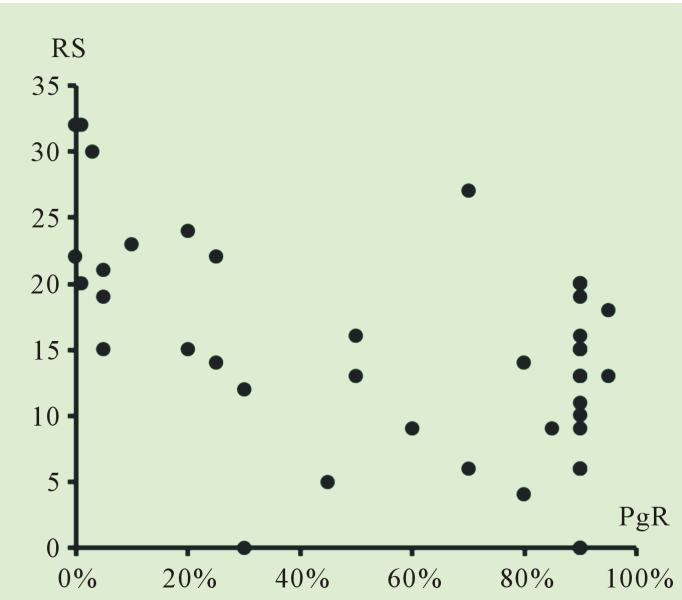

Figure 1. Correlation of Oncotype DX RS with pgR statu. Correlation coefficient $=0.50, \mathrm{P}<0.01$.

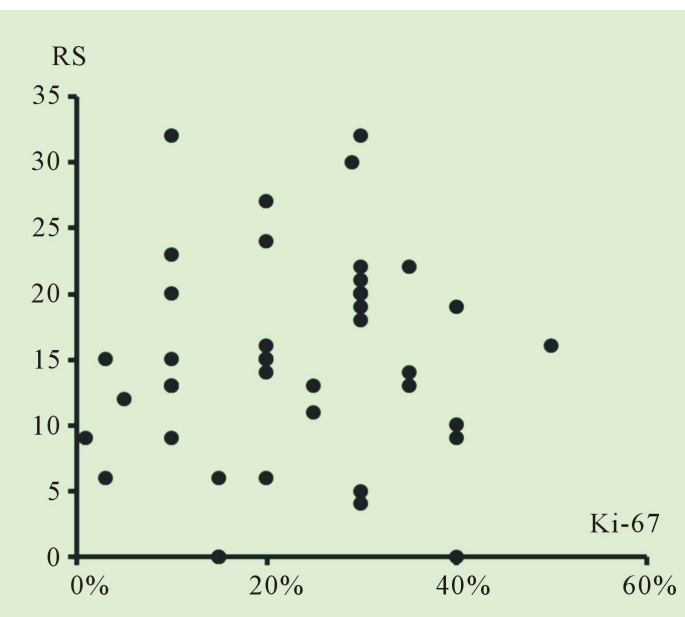

Figure 2. Correlation of Oncotype DX RS with Ki-67 (local). Correlation coefficient $=0.08, \mathrm{P}=0.30$.

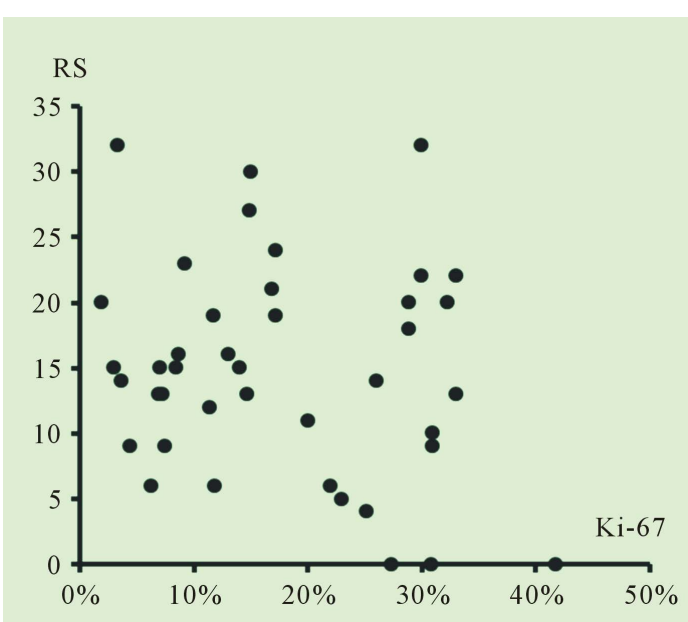

Figure 3. Correlation of Oncotype DX RS with Ki-67 (central). Correlation coefficient $=0.09, \mathrm{P}=0.28$. 


\section{Discussion}

The results of the present study showed that the newly proposed luminal subtypes with adjunctive reassessment of Ki-67 by central review was associated with a higher concordance rate between the luminal subtypes and risk groups of RS than that of previously proposed luminal subtypes. In this study, by introducing the newly proposed luminal subtypes and reassessment of Ki-67 by central review, the concordance rate between the luminal $\mathrm{A}$ and the low-risk group and between the luminal B and the intermediate to high risk groups improved from $72.7 \%$ to $100 \%$ and $40.0 \%$ to $53.6 \%$, respectively. We also showed that there was a significant correlation between PgR status and Oncotype RS. However, there was no significant correlation between Ki-67 status as assessed by either a local pathologist or central review and the Oncotype RS.

Previous studies have demonstrated that lower expression of PgR has been associated with higher Oncotype DX RS [25] [26]. Moreover, another paper reported that a strong correlation existed between the Ki-67 value and Oncotype DX RS, especially in tumors with a Ki-67 value $\geq 25 \%$. They also found that the likelihood of a tumor with a Ki-67 value $\geq 25 \%$ having an intermediate or high Oncotype RS is $>90 \%$ and these patients may benefit from adjuvant chemotherapy [27]. In this study, there was a statistically significant correlation between PgR and Oncotype DX RS, but there was no significant correlation between Ki-67 status as assessed by either a local pathologist or central review and Oncotype RS.

The International Ki-67 in Breast Cancer Working Group published their recommendations for Ki-67 assessment in breast cancer in 2011. However, these recommendations included no established quality assurance schemes to ensure that the procedures for Ki-67 analysis in one laboratory lead to comparable scores in others, and this article did not recommend standardizing an evaluation method of Ki-67 [9]. Thus, the direct application of specific cut-off rates for comparison must be considered unreliable unless the analyses are performed in a high-volume laboratory with its own reference data. Our previous study analyzed 287 primary breast cancer patients who underwent preoperative CNB to compare the concordance rates for assessing Ki-67 status evaluated either by automated or central/local pathology assessment and showed that central review and the use of an automated analyzer can improve the accuracy of Ki-67 assessment. We then confirmed the necessity of a standardized evaluation method for Ki-67 expression in breast cancer to overcome the disadvantages of variable counting methods and measurement sites [23] [24].

Current immunohistochemical markers do not allow accurate prediction of the risk of recurrence, and improvements are required to clearly identify which women are at sufficiently low risk to be able to safely avoid the use of chemotherapy. A previous study reported that the Oncotype DX RS provided additional prognostic information regarding distant recurrence beyond classical clinicopathologic factors. However, its cost is a barrier for its use in many other countries. It is therefore important to select appropriate patients for the Oncotype DX test.

A major limitation of this study is that it is a retrospective chart review with a small sample size, non-randomized, and limited to a single oncology center. Despite these limitations, this approach may have wide applicability and improve clinical information when selecting appropriate patients for the Oncotype DX test.

\section{Conclusion}

The newly proposed luminal subtypes with adjunctive reassessment of Ki-67 by central review appears to improve the concordance rate-especially between luminal A and low RS category. However, verification in a larger trial is needed to assess whether this approach can reduce the breast cancer recurrence; our preliminary findings indicate a low cost way to select low risk patients for whom adjuvant chemotherapy could be avoided.

\section{Acknowledgements}

The authors would like to thank Enago (www.enago.jp) for the English language review. This study was presented in part at the 2015 Global Breast Cancer Conference (poster).

\section{Competing Interests}

The authors declare no competing interests. 


\section{Disclosure}

The authors declare no conflicts of interest.

\section{References}

[1] Early Breast Cancer Trialists’ Collaborative Group (EBCTCG) (2005) Effects of Chemotherapy and Hormonal Therapy for Early Breast Cancer on Recurrence and 15-Year Survival: An Overview of the Randomised Trials. Lancet, 365 , 1687-1717. http://dx.doi.org/10.1016/S0140-6736(05)66544-0

[2] Prat, A., Pineda, E., Adamo, B., et al. (2015) Clinical Implications of the Intrinsic Molecular Subtypes of Breast Cancer. Breast, 24, S26-S35. http://dx.doi.org/10.1016/j.breast.2015.07.008

[3] Lopez, F., Belloc, F., Lacombe, F., et al. (1991) Modalities of Synthesis of Ki-67 Antigen during the Stimulation of Lymphocytes. Cytometry, 12, 42-49. http://dx.doi.org/10.1002/cyto.990120107

[4] Urruticoechea, A., Smith, I.E. and Dowsett, M. (2005) Proliferation Marker Ki-67 in Early Breast Cancer. Journal of Clinical Oncology, 23, 7212-7220. http://dx.doi.org/10.1200/JCO.2005.07.501

[5] Trihia, H., Murray, S., Price, K., et al. (2003) Ki-67 Expression in Breast Carcinoma: Its Association with Grading Systems, Clinical Parameters, and Other Prognostic Factors-A Surrogate Marker? Cancer, 97, 1321-1331. http://dx.doi.org/10.1002/cncr.11188

[6] Spyratos, F., Ferrero-Pous, M., Trassard, M., et al. (2002) Correlation between MIB-1 and Other Proliferation Markers: Clinical Implications of the MIB-1 Cutoff Value. Cancer, 94, 2151-2159. http://dx.doi.org/10.1002/cncr.10458

[7] Cheang, M.C., Chia, S.K., Voduc, D., et al. (2009) Ki67 Index, HER2 Status, and Prognosis of Patients with Luminal B Breast Cancer. Journal of the National Cancer Institute, 101, 736-750. http://dx.doi.org/10.1093/jnci/djp082

[8] Bouzubar, N., Walker, K.J., Griffiths, K., et al. (1989) Ki67 Immunostaining in Primary Breast Cancer: Pathological and Clinical Associations. British Journal of Cancer, 59, 943-947. http://dx.doi.org/10.1038/bjc.1989.200

[9] Dowsett, M., Nielsen, T.O., A’Hern, R., et al. (2011) Assessment of Ki67 in Breast Cancer: Recommendations from the International Ki67 in Breast Cancer Working Group. Journal of the National Cancer Institute, 103, 1656-1664. http://dx.doi.org/10.1093/jnci/djr393

[10] Dowsett, M., Cuzick, J., Wale, C., et al. (2010) Prediction of Risk of Distant Recurrence Using the 21-Gene Recurrence Score in Node-Negative and Node-Positive Postmenopausal Patients with Breast Cancer Treated with Anastrozole or Tamoxifen: A Trans ATAC Study. Journal of Clinical Oncology, 28, 1829-1834. http://dx.doi.org/10.1200/JCO.2009.24.4798

[11] Paik, S., Tang, G., Shak, S., et al. (2006) Gene Expression and Benefit of Chemotherapy in Women with Node-Negative, Estrogen Receptor-Positive Breast Cancer. Journal of Clinical Oncology, 24, 3726-3734. http://dx.doi.org/10.1200/JCO.2005.04.7985

[12] Chen, C., Dhanda, R., Tseng, W.-T., et al. (2013) Evaluating Use Characteristics for the Oncotype Dx 21-Gene Recurrence Score and Concordance with Chemotherapy Use in Early-Stage Breast Cancer. Journal of Oncology Practice/ American Society of Clinical Oncology, 9, 182-187. http://dx.doi.org/10.1200/JOP.2012.000638

[13] Sparano, J.A. and Paik, S. (2008) Development of the 21-Gene Assay and Its Application in Clinical Practice and Clinical Trials. Journal of Clinical Oncology, 26, 721-728. http://dx.doi.org/10.1200/JCO.2007.15.1068

[14] Tang, G., Cuzick, J., Costantino, J.P., et al. (2011) Risk of Recurrence and Chemotherapy Benefit for Patients with Node-Negative, Estrogen Receptor-Positive Breast Cancer: Recurrence Score Alone and Integrated with Pathologic and Clinical Factors. Journal of Clinical Oncology, 29, 4365-4372.

[15] Mamounas, E.P., Tang, G., Fisher, B., et al. (2010) Association between the 21-Gene Recurrence Score Assay and Risk of Locoregional Recurrence in Node-Negative, Estrogen Receptor-Positive Breast Cancer: Results from NSABP B-14 and NSABP B-20. Journal of Clinical Oncology, 28, 1677-1683. http://dx.doi.org/10.1200/JCO.2009.23.7610

[16] Dowsett, M., Ebbs, S.R., Dixon, J.M., et al. (2005) Biomarker Changes during Neoadjuvant Anastrozole, Tamoxifen, or the Combination: Influence of Hormonal Status and HER-2 in Breast Cancer-A Study from the IMPACT Trialists. Journal of Clinical Oncology, 23, 2477-2492. http://dx.doi.org/10.1200/JCO.2005.07.559

[17] Harris, L., Fritsche, H., Mennel, R., et al. (2007) American Society of Clinical Oncology 2007 Update of Recommendations for the Use of Tumor Markers in Breast Cancer. Journal of Clinical Oncology, 25, 5287-5312. http://dx.doi.org/10.1200/JCO.2007.14.2364

[18] National Comprehensive Cancer Network Practice Guidelines in Oncology. Breast Cancer (Version v2.2011). http://www.NCCN.org

[19] Aebi, S., Davidson, T., Gruber, G., et al. (2010) Primary Breast Cancer: ESMO Clinical Practice Guidelines for Diag- 
nosis, Treatment and Follow-Up. Annals of Oncology, 21, v9-v14. http://dx.doi.org/10.1093/annonc/mdq159

[20] Goldhirsch, A., Wood, C. and Coates, A.S. (2011) Strategies for Subtypes-Dealing with the Diversity of Breast Cancer: Highlights of the St Gallen International Expert Consensus on the Primary Therapy of Early Breast Cancer 2011. Annals of Oncology, 22, 1736-1747. http://dx.doi.org/10.1093/annonc/mdr304

[21] Goldhirsch, A., Ingle, J.N., Gelber, R.D., et al. (2009) Thresholds for Therapies: Highlights of the St Gallen International Expert Consensus on the Primary Therapy of Early Breast Cancer 2009. Annals of Oncology, 20, 1319-1329. http://dx.doi.org/10.1093/annonc/mdp322

[22] Prat, A., Cheang, M.G.U., Martín, M., et al. (2013) Prognostic Significance of Progesterone Receptor-Positive Tumor Cells within Immunohistochemically Defined Luminal A Breast Cancer. Journal of Clinical Oncology, 31, 203-209. http://dx.doi.org/10.1200/JCO.2012.43.4134

[23] Mizuno, Y., Natori, T., Takeda, N., et al. (2012) The Reliability of Assessment of Ki-67 Expression on Core Needle Biopsy and the Surgical Specimens of Invasive Breast Cancer: Comparison of Local Pathologists' Assessment and Central Review. Journal of Cancer Therapy, 3, 841-845. http://dx.doi.org/10.4236/jct.2012.325107

[24] Mizuno, Y., Fuchikami, H., Takeda, N., et al. (2014) Standardized Assessment of Ki-67 in Breast Cancer Patients Using Virtual Slides and an Automated Analyzer in Comparison to Central/Local Pathological Assessments. Journal of Cancer Therapy, 5, 141-146. http://dx.doi.org/10.4236/jct.2014.52017

[25] Flanagan, M.B., Dabbs, D.J., Brufsky, A.M., et al. (2008) Histopathologic Variables Predict Oncotype DX Recurrence Score. Modern Pathology, 21, 1255-1261. http://dx.doi.org/10.1038/modpathol.2008.54

[26] Tang, P., Wang, J., Hicks, D.G., et al. (2010) A Lower Allred Score for Progesterone Receptor Is Strongly Associated with a Higher Recurrence Score of 21-Gene Assay in Breast Cancer. Cancer Investigation, 28, 978-982. http://dx.doi.org/10.3109/07357907.2010.496754

[27] Sahebjam, S., Aloyz, R., Pilavdzic, D., et al. (2011) Ki 67 Is a Major, But Not the Sole Determinant of Oncotype DX Recurrence Score. British Journal of Cancer, 105, 1342-1345. http://dx.doi.org/10.1038/bjc.2011.402 